Meta

Journal des traducteurs

Translators' Journal

\title{
À l'écoute de la langue vivante
}

\section{Robert Dubuc}

Volume 25, numéro 2, juin 1980

URI : https://id.erudit.org/iderudit/002630ar

DOI : https://doi.org/10.7202/002630ar

Aller au sommaire du numéro

Éditeur(s)

Les Presses de l'Université de Montréal

ISSN

0026-0452 (imprimé)

1492-1421 (numérique)

Découvrir la revue

Citer cet article

Dubuc, R. (1980). À l'écoute de la langue vivante. Meta, 25(2), 249-253.

https://doi.org/10.7202/002630ar

Ce document est protégé par la loi sur le droit d'auteur. L’utilisation des services d'Érudit (y compris la reproduction) est assujettie à sa politique d'utilisation que vous pouvez consulter en ligne.

https://apropos.erudit.org/fr/usagers/politique-dutilisation/
Cet article est diffusé et préservé par Érudit.

Érudit est un consortium interuniversitaire sans but lucratif composé de l’Université de Montréal, l'Université Laval et l'Université du Québec à Montréal. Il a pour mission la promotion et la valorisation de la recherche. https://www.erudit.org/fr/ 


\section{PROBLÈMES ET SOLUTIONS}

\section{À L'ÉCOUTE DE LA LANGUE VIVANTE}

C'est aujourd'hui un truisme de dire que la langue est un organisme vivant. Pourtant nos vieux schèmes fixistes restent toujours à l'œuvre dans quelque coin de notre univers mental, si bien qu'on reste en général assez prévenu face au changement de ses habitudes langagières. Témoin, cette résistance au féminin dans les noms de profession qui représente une sorte de crispation de l'usager en face de l'adaptation de ses habitudes de langage à une donnée de l'évolution sociale.

À côté de cette résistance peuvent se développer des engouements pour la nouveauté. La vogue d'un mot comme créneau, d'un affixe comme mini est essentiellement un phénomène de mode qui a peu à voir en fin de compte avec les besoins réels d'expression des usagers. Mais le facteur mode reste un ingrédient de la mystérieuse alchimie qui gouverne l'évolution du vocabulaire d'une langue. Pourtant il n'y a pas que la mode; il y a les besoins réels ou imaginés, la crainte de choquer, celle de ne pas être compris, le désir de faire de l'effet. Et en filigrane, derrière des motifs consciemment identifiables, il y a la structure de la langue elle-même qui exerce son influence en appliquant les principes d'économie - le moins de mots pour dire le plus possible - et les «règles» de sa morphologie.

C'est donc dans ce décor complexe que se situe la néologie d'aujourd'hui et c'est dans ce décor qu'il faut l'étudier et la voir à l'œuvre.

\section{Comment se présente la néologie d'aujourd'hui en français}

Pour étudier la néologie en français contemporain nous avons réuni au hasard un échantillon de deux cents néologismes : cent pour la langue courante; cent pour la langue technique. C'est à partir de cet échantillon - avec ses limites et ses risques de gauchissement - que nous avons fondé nos observations.

La première constatation que l'on peut faire c'est que le français contemporain suit fondamentalement le schème classique de la formation des mots. Ceux qui veulent épousseter leur mémoire pourront se reporter au chapitre VII 


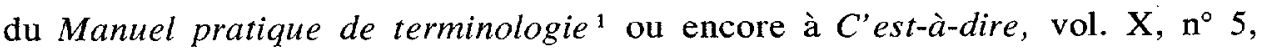
1978 pour avoir un exposé complet — quoique sommaire — des procédés de formation des mots en français.

La seconde constatation, c'est que la langue commune et la langue technique diffèrent sensiblement dans les procédés qu'elles appliquent respectivement.

Au chapitre de la formation indirecte, la langue commune crée $40 \%$ de ses termes par les divers procédés d'extension de sens; la langue technique n'y a recours que pour $8 \%$ seulement.

Par contre, lorsqu'il s'agit de formation directe, la balance est renversée : la langue technique fait appel à la composition pour $61 \%$ de ses néologismes, contre $20 \%$ pour la langue courante.

Toutefois la dérivation est à peu près également mise à contribution aux deux niveaux, soit $29 \%$ pour la langue courante et $27 \%$ pour la langue technique.

Tous les autres procédés: assimilation, changement de catégorie grammaticale, dérivation régressive, télescopage, acronymisation et emprunt ne comptent que pour $11 \%$ des créations en langue courante et $4 \%$ en langue technique.

La part congrue faite à l'emprunt étonne beaucoup. On a parfois l'impression d'une véritable marée d'emprunts, en particulier dans la langue technique. Or, sous réserve des erreurs d'échantillonnage, l'emprunt est plus fréquent dans la langue commune $(9 \%)$ que dans la langue technique où il ne compte que pour $3 \%$ des néologismes.

Quelques procédés semblent un peu mis en veilleuse : par exemple, on se résigne difficilement à emprunter aux autres disciplines, à l'exception de la gestion qui emprunte beaucoup au langage militaire : cadres, état-major, logistique.

La dérivation régressive apparaît à peu près absente du paysage si on excepte calfeutre tiré de calfeutrer. Par contre, on trouve quelques cas de changement de catégorie grammaticale : les impayés, les postés qui passent du participe adjectif au nom.

\section{Comment on applique les procédés classiques de formation des mots}

En ce qui concerne le vocabulaire technique, la composition doit principalement nous retenir puisqu'elle compte à elle seule pour plus de $60 \%$ des créations.

Cette vogue de la composition s'explique en partie par le goût des techniciens pour les termes quasi-définitions. On souhaite que l'étiquette donnée à une réalité nouvelle en donne aussi un peu la signification : d'où la grande mode des compositions à éléments signifiants : mots pleins coordonnés : sac-palette, mot-

1. Robert Dubuc, Manuel pratique de terminologie, Montréal, Linguatech, 1978. Comité de linguistique, C'est-à-dire, Montréal, Radio-Canada, vol. X, n 5, 1978. 
souche avec complément déterminatif : pochette d'emballage, tête de cerclage à lancement automatique.

On pratique aussi la composition par éléments signifiants : racines latines, grecques ou françaises. Ce qui caractérise l'application de ce procédé, c'est sa grande liberté. Les règles de la morphologie classique contre l'hybridation des racines sont complètement enfoncées: on allie volontiers latin et grec : hypocalorique; grec et français: télécopie, télésurveillance (monitoring); latin et français : vidéotexte.

La composition morphologique contemporaine se distingue encore par la création de nouveaux affixes, souvent sur le modèle des racines anciennes.

Par exemple: $\quad$ auto - à l'origine racine grecque signifiant soi-même - lié à l'idée d'automaticité, devient une abréviation du mot automobile dans les créations suivantes : auto-radio, auto-route, auto-parc, auto-école, etc.

télé - à l'origine racine grecque signifiant au loin, à distance, devient une abréviation de télévision dans des formations comme téléroman, téléjournal, téléthéâtre, téléaste.

cyclo - élément grecque signifiant cercle, cycle, devient le symbole de la bicyclette dans cyclo-tourisme, cyclopousse, cyclomoteur, piste cyclable.

thèque - suffixe grec désignant un lieu de rangement; s'applique à des établissements de prêts: ludothèque, joujouthèque, ou même à des lieux d'activité: discothèque.

Parmi ces créations, il faut faire un sort spécial à mini qui, la mode aidant, a connu une fortune tout à fait extraordinaire. Cet affixe conserve sans doute une parenté avec l'adjectif latin minimum, et il a transposé en français l'idée de très petit, très peu, contenue dans l'adjectif latin. Dans le sillage de la minijupe, le mot a fait fortune tant dans la langue technique, avec mini-ordinateur, miniprogramme, mini-métro, mini-cassette, que dans la langue courante avec miniappartement, mini-budget, mini-bouteille, etc.

Il est même devenu substantif : «Réduire tout à l'échelle du mini» et adjectif : «Un moteur diesel vraiment mini.» succès.

Il s'est en outre suscité un contraire : maxi, qui connaît lui aussi un certain

La vogue de la composition a aussi favorisé deux procédés de formation empruntés à l'anglais, mais acclimatés en français : le téléscopage et l'acronymisation.

Le premier, beaucoup plus fécond que le second, consiste à former un mot avec des parties d'autres mots qu'on soude ensemble. C'est ce procédé qui nous a donné informatique (information automatique), alco-essence (alcool et es- 
sence), motel (moteur-hôtel, de l'anglais), et qui est en train de créer un nouveau suffixe «ique» avec le sens de «technique moderne»: mercatique, créatique, bureautique.

L'autre procédé consiste à créer un mot, soit avec les initiales, soit avec des syllabes de plusieurs mots pour former un acronyme. ADAC - ADAV sont les prototypes de ces créations en français d'après le modèle anglais qui a donné radar, laser, maser, etc.

Quant à la dérivation par suffixe, il faut noter une certaine polarisation autour des suffixes -iser et -isation pour les verbes et substantifs d'action: dramatiser, dramatisation, politiser, politisation, etc.

Ce modèle de dérivation est extrêmement fréquent. Autre trait significatif, la désignation des machines par le suffixe féminin -euse - visseuse, hausseuse, sertisseuse, emballeuse, tandis que le suffixe -eur désigne plutôt des appareils: disjoncteur, générateur, présentateur de sacs, etc. Il convient de noter aussi la vogue du suffixe -iste pour désigner un agent: croisièriste, excursionniste, forfaitiste, plagiste, voyagiste. Ce qui n'exclut pas le suffixe -eur utilisé dans le même sens : lotisseur, aménageur, randonneur ${ }^{2}$, etc.

\section{Attitude à adopter}

Face à cette floraison, quelle attitude le terminologue et autres outilleurs du langage doivent-ils adopter?

La première attitude à conseiller en est une de prudence. Il ne faut pas créer sans besoin et risquer de mettre en circulation des termes parallèles. JeanClaude Corbeil a relevé plus de 14 termes synonymes employés par les fabricants de réfrigérateurs pour désigner le compartiment à ceufs. Une telle abondance est une forme de gaspillage d'énergie. Avant de créer un mot, il faut s'assurer dans toute la mesure du possible qu'il n'en existe pas déjà un.

Deuxièmement, il faut respecter les modes de formation des mots en usage dans la langue qu'on utilise. Il faut donc connaître ces procédés et savoir les appliquer. L'analyse morphologique des néologismes qu'on rencontre peut servir utilement à cette fin.

Troisièmement, il faut tirer parti de toutes les ressources morphologiques de la langue. Savoir, par exemple, qu'il n'y a pas que les suffixes eur, -euse, iser, -isation; savoir que la composition n'est pas la seule façon de créer un mot nouveau, qu'on peut notamment avoir recours à l'extension sémantique, à l'emprunt interdisciplinaire et à la dérivation régressive.

Quatrièmement, se rappeler qu'un terme doit être une étiquette et non une définition. À cet égard, il faut se méfier de l'abus de la composition. La loi de l'économie exige qu'un terme soit bref dans toute la mesure du possible, sans quoi l'expression s'alourdit et devient embarrassée.

2. Exemples empruntés à Charles Dupont, Vocabulaire du tourisme, Montréal, Linguatech, 1979, $149 \mathrm{p}$. 
Cinquièmement, rapprocher la langue technique de la langue commune en pratiquant davantage l'extension sémantique et l'analogie, soit de forme, soit de fonction.

Sixièmement, se tranquilliser un peu la conscience face à l'emprunt aux langues étrangères. Au Canada, nous sommes sursensibilisés face à l'emprunt. Cette prudence s'impose sans doute en raison de la précarité de notre situation. Encore faudrait-il que cette prudence ne dégénère pas en psychose, comme c'est le cas lorsque certains s'insurgent contre week-end, stop ou gag. Il ne sert à rien de vouloir déraciner des emprunts vieux de cinquante ou soixante ans. Sachons mieux employer nos énergies.

\section{Conclusion}

En matière de néologie, la meilleure arme du terminologue et du traducteur, c'est encore le bain de langue vivante que constitue la lecture critique des revues françaises, tant spécialisées que celles destinées au grand public. Dans la lecture de ces revues, il faut être particulièrement attentif aux nouveautés quelles qu'elles soient : sémantiques, grammaticales ou autres.

Voici un exemple d'observations recueillies dans quelques numéros de la revue Usine nouvelle.

On trouve équipement de télétexte pour rendre word processing equipment terminal intelligent (doté d'une mémoire)

reprographe : (appareil qui reproduit une image sur tube cathodique)

système de recherche de personne (paging system)

robot de lavage (automatic car wash)

décideur-décisionnaire (decision makers)

péritéléphonie (qui comprend télécopie, vidéotexte, télésurveillance)

télésurveillance (pour monitoring)

caloduc : récupérateur de chaleur

temporisateur : minuterie programmable

héliothermicien : spécialiste de l'énergie solaire

postés : pour shift workers

agroalimentaire : là où on dirait simplement alimentaire

électroacoustique : là où le commerce emploie audio

le mot profession pour désigner l'industrie, c'est-à-dire l'ensemble des industriels. Ex. : La profession de l'électroménager

l'élimination du mot «bulldozer» au profit de bouteur

produit grand-public : consumer's product.

Parce que rien n'est jamais acquis et que tout ce qui vit est en mouvement, il faut se tenir à l'écoute attentive de la langue vivante partout où elle se parle.

Robert Dubuc

BIBLIOGRAPHIE

GILBERT, Pierre, Dictionnaire des mots nouveaux, Paris, Hachette-Chou, 1971, 570 p.

Usine nouvelle, 15, rue Bleue, 75009, Paris, Édition hebdomadaire. 